\title{
COMMISSION 15: PHYSICAL STUDY OF COMETS, MINOR PLANETS, AND METEORITES
}

\section{(L'ETUDE PHYSIQUE DES COMETES, DES PETITES PLANETES ET DES METEORITES)}

\author{
PRESIDENT: Vincenzo Zappalá \\ VICE-PRESIDENT: H. Uwe Keller \\ ORGANIZING COMMITTEE: M. Bailey, R. P. Binzel, M. T. Capria, \\ P. D. Feldman, J. Fernández, C.-I. Lagerkvist, A.-C. Levasseur-Regourd, \\ K. Meech, J. Watanabe \& R. M. West
}

\section{INTRODUCTION}

The present report of Commission 15 has been, as usual, prepared primarily by the chairpersons of the two working groups. E. Tedesco wrote the section about Asteroids and Meteorites, with the assistance of A. Cellino, G. Consolmagno and C.-I. Lagerkvist. W. F. Huebner prepared the section about Comets, with the assistance of $\mathbf{J}$. Benkhoff, H. Boehnhardt, J. Brandt, M. T. Capria, A. Cochran, G. Cremonese, M. Duncan, W. Huntress, H. Levison, and G. P. Tozzi. Moreover, the whole document has been assembled by $K$. Muinonen, who did the final editing, to merge the two reports and fit the document into the allotted space. Material taken from both major areas regarding the relationship between comets and asteroids has been combined into a single section.

Scientific activity in the field has been very intense in the past three years, and led to publication of a very large number of papers. This is a convincing proof of the present good health of Planetary Sciences, also for what concerns the Minor Bodies. On the other hand, such an impressive amount of papers constitutes a problem when an attempt at summarizing the ongoing activity is made, like in the present case. For this reason, and following the approach used in the last Commission report, the present document does not include a comprehensive bibliography, since this would have taken by itself a major fraction of the allotted pages. We have therefore prepared a selective review of results, but giving no list of references. References are cited in the text by year, and in the case of the asteroids also with a topic number and a sequential number indicating the location of each paper in a bibliographic database which has been created by the Asteroid Working Group. Thus (1997, T06-011) refers to the 11th paper (published in 1997) in 6th topic defined in the database. The comprehensive list of references (including separately the asteroid bibliographic database described above) is available at the commission's WWW page, which can be reached as a link from the IAU's home page (http://www.iau.org).

\section{ASTEROIDS}

In this section, papers related to asteroid physical studies and their connections with meteorites are briefly summarized. This report is based upon a compilation of 317 refereed papers. Using a format similar to that used in the previous transactions, the material is organized into a series of 12 arbitrary categories. In many cases a given paper could have been assigned to one of several different categories. The final choice was at the whim of the database compiler (E. Tedesco).

\subsection{Size distributions, diameters, and masses}

Size distributions Jedicke and Metcalfe (1998, T01-007) present a model-independent analytical method for debiasing the four-dimensional $(a, e, i, H)$ distribution obtained in any 
asteroid observation program and apply the technique to results obtained with the $0.9-\mathrm{m}$ Spacewatch telescope. They found that the absolute magnitude - frequency distribution cannot be represented by a single power law $10^{\alpha H}$ in any region of the asteroid belt. They were able to define broad ranges in $H$ in each part of the belt where $\alpha$ was nearly constant and found that within these ranges of $H$ the slope does not correspond to the value of 0.5 expected for an equilibrium cascade in self-similar collisions (Dohnanyi 1971). They found that the value of $\alpha$ varies with absolute magnitude and shows a "kink" in all regions of the belt for $H \sim 13$.

Evans et al. (1998, T01-008) report on a study in which they extracted serendipitous observations of 96 moving objects from examination of 28,460 selected Wide-Field and Planetary Camera 2 long exposures from 1994, 1995, and early 1996 Hubble Space Telescope images. Most of the objects are new, as they are too faint to show up in ground-based surveys. They determined distances for many of these objects using the parallax contribution to the trail shapes. Based on these distances, constraints on the orbits, and photometry of the trails $(16<V<24)$, most of the moving objects appear to be small, main-belt asteroids a few $\mathrm{km}$ in diameter. A few are known objects, three are potential Mars crossers. Modern wide-field CCD surveys detect asteroids nearly as faint as these $(V<21)$, but the corresponding absolute magnitudes are uncertain unless their orbits have been established.

The detected objects span the absolute magnitude range $13.6<H<19.3$. They report that the statistics of the detections imply a reservoir of $3.1( \pm 0.6) \times 10^{5}$ such asteroids within $25 \mathrm{deg}$ of the ecliptic. They find that the slope of the cumulative distribution of absolute magnitudes follows a power law $N \sim H^{0.2}$ to $N \sim H^{0.3}$ over this absolute magnitude range in the three distance ranges defined by the Palomar-Leiden survey and note that these are significantly shallower slopes than those inferred by the Palomar-Leiden survey or extrapolated from population studies of larger asteroids.

Diameters Hestroffer and Mignard (1997, T01-003) show how observations of an extended object using a periodic grid may also provide information on its size and on the distribution of light over its surface. They discuss the method and use Hipparcos observations of 1 Ceres to illustrate its application.

Drummond et al. (1998, T01-009) reported adaptive optics observations of Ceres and Vesta obtained with the 1.5-m telescope at the Starfire Optical Range of the USAF Phillips Laboratory located near Albuquerque, NM and analyzed with a new reconstruction technique, called parametric blind deconvolution. The triaxial ellipsoid dimensions and rotational pole (with a two-fold ambiguity) of Ceres were obtained from 17 images and found to be in excellent agreement with previous results from stellar occultation and adaptive optics observations. The results for Vesta yield dimensions and a rotational pole in reasonable agreement with the Hubble Space Telescope's results (Thomas et al. 1997, T06-018).

Masses Hilton et al. (1996, T01-001) investigated the prospects for determining asteroid masses by integrating the orbits of 4583 main-belt asteroids for a period of 57 years and searching for asteroid-asteroid encounters from which it might be possible to determine masses for the 23 largest and 11 smaller asteroids. They found 460 such encounters and examined ten in detail. They present a list of 34 asteroids for which astrometric observations stand the greatest chance of determining a mass for the larger asteroid and conclude that targeting the asteroids in this list would have a substantial impact on our knowledge of asteroid masses. Su (1999, T01-011) discussed the determination of asteroid masses via space missions to asteroids.

New mass determinations were published for six different asteroids (five of which are in the Hilton et al. (1996) list mentioned above): 1 Ceres, 2 Pallas, 4 Vesta, 11 Parthenope, 15 Eunomia, and 20 Massalia.

Results for Ceres were published by Viateau and Rapaport (1998, T01-005) and by Hilton (1999, T01-010). These authors find a mass of $4.39( \pm 0.04) \times 10^{-10}$ and $4.759( \pm 0.023)$ $\times 10^{-10} \mathrm{M}_{\odot}$, respectively, leading Viateau and Rapaport to conclude that the mass of Ceres 
recommended by the IAU should be decreased by nearly $5 \%$. In these same papers Viateau and Rapaport also published a mass for 11 Parthenope while Hilton also published masses for 2 Pallas, and 4 Vesta. The Viateau and Rapaport results utilized Hipparcos data while that of Hilton was based upon a total of 59,258 optical and two radar observations used to fit the ephemerides. Hilton's mass determinations were done in conjunction with the publishing of a new set of ephemerides for 15 of the largest asteroids, covering the period from 1800 through 2100, for use in the Astronomical Almanac.

Hilton (1997, T01-004) published a mass for 15 Eunomia and Bange (1998, T01-006) one for 20 Massalia. Bange's results made use of Hipparcos data.

Wilson et al. (1999, T01-012), using the Martian satellites Phobos and Deimos and main-belt asteroids $243 \mathrm{Ida}$ and 253 Mathilde, all of whose densities are known to within about $10 \%$ from spacecraft flybys, note that all of these asteroids are significantly less dense than most members of the classes of meteorites identified as being compositionally most nearly similar to them on the basis of spectral characteristics. They discuss two processes that can act, independently or in concert, to produce these low bulk densities. One of these processes is the result of one or more impact events and can affect any asteroid, whereas the other can occur only for certain classes of small asteroids which have undergone aqueous alteration.

\subsection{Photometry, shapes, disk-resolved images, and rotation}

Lightcurve photometry continues to be an active area of asteroid observations and is the basis for most of the research described in this chapter. Wisniewski et al. (1997, T02-020) published lightcurve data for 125 asteroids. Contributions in this field were also made by Florczak et al. (1997, T02-025), Denchev et al. (1998, T02-036) and Piironen et al. (1998, T02-031). Asteroid 15 Eunomia was studied in detail with near-infrared reflected and thermal emitted lightcurves by Reed et al. (1997, T02-019). Photometry of NEOs were done by Pravec et al. (1996, T02-007) and several NEOs were studied in greater detail by Magnusson et al. (1996, T02-002); Mottola et al. (1997, T02-014); Nakamura and Fuse (1998, T02-037); and Kryszczy et al. (1999, T02-041). Donnison and Wiper (1999, T02-040) used these data in a statistical study of asteroid rotation rates.

Pole determinations were published for a total of 50 asteroids by Michalowski (1996, T02-003), Kryszczynska et al. (1996, T02-005), Blanco and Riccioli (1998, T02-033), and Denchev et al. (1998, T02-036). Skoglöf et al. (1996, T02-009) made a theoretical study of the evolution of the obliquities for ten asteroids. The Hipparcos solar system objects catalogues were published by Hestroffer et al. (1998, T02-030). Lagerkvist et al. (1996, T02045) published the fourth update of the Asteroid Photometric Catalogue. Hernius et al. (1997, T02-010) made a study of the size distribution of main-belt asteroids and Lagerkvist and Lagerros (1997, T02-046) investigated the number density of main-belt asteroids.

Explicit studies of different asteroid groupings were made by Slivan et al. (1996, T02006) of the Koronis family, by Dahlgren et al. (1998, T02-035; 1999, T02-042) of the Hilda group and by Lagerkvist et al. (1998, T02-032) of asteroids of type M.

Kristensen and Gammelgaard (1997, T02-011) continued their observations of $51 \mathrm{Ne}-$ mausa studying the phase dependences. Phase curves obtained with the Carlsberg meridian instrument were published by Piironen et al. (1997, T02-12). Asteroid observations at low phase angles were made by Shevchenko et al. (1997, T02-26).

Muinonen introduced the Gaussian shape hypothesis for asteroids and comets (1998, T02-028) and it was used by Muinonen and Lagerros (1998, T02-029) for inversion of the shape statistics of small solar system bodies. The Hubble Space Telescope was used in several studies for imaging of asteroids by Zellner et al. (1997, T02-021), Binzel et al. (1997, T02-22), and Storrs et al. (1999, T02-039). 


\subsection{Radar, millimeter, radiometry, polarimetry, and occultation studies}

Radar Radar observations or modeling studies were published for three main-belt asteroids (1 Ceres, 2 Pallas, and 4 Vesta) by Mitchell et al. (1996, T03-002) and for three near-Earth asteroids (433 Eros, 2063 Bacchus, and 4769 Castalia) by Mitchell et al. (1998, T03-014), Benner et al. (1999, T03-016) and Hudson et al. (1997, T03-005), respectively.

Observations of the main-belt asteroids help to characterize their surface properties. For example, the authors note that: "Given a porosity of $45 \%$, the specific gravities of the surface materials on Ceres and Pallas would be $\sim 2.3$ and $\sim 3.0 \mathrm{~g} \mathrm{~cm}^{-} 3$, respectively, which would be consistent with (1) the presence of an additional silicate component on Pallas' surface (as inferred from spectroscopic observations) and (2) recent mass estimates, which suggest a higher mean (volume-averaged) density for Pallas than for Ceres. "

Mitchell et al. (T03-014) used new analysis techniques on Goldstone radar data obtained during the asteroid's close approach in 1975 to constrain the shape of 433 Eros. They concluded, in part, that the pole-on silhouette of the Eros is shaped like a kidney bean, although they could not exclude shapes with more than one large concavity. By correlating variations in the pyroxene-olivine ratio over Eros' surface inferred from visual and infrared observations with their shape information, they found that the side with concavities is relatively pyroxene-rich compared with the more rounded opposing side.

Benner et al. reported on radar observations of 2063 Bacchus obtained at Goldstone in March 1996. Inversion of delay-Doppler images, cw spectra, and optical lightcurves obtained at Ondrejov Observatory were used to derive lower and upper bounds on the degree of bifurcation. They found that both shape models have a prominent central concavity, modestly asymmetric shapes, and similar physical dimensions, spin vectors, and radar and optical geometric albedos. Adopting the more conservative single-lobe shape model as their working model they computed a pole orientation, sidereal rotation period, and size for Bacchus, for which they concluded retrograde rotation is likely.

Hudson et al. combined lightcurve observations of 4769 Castalia with a 167-parameter 3-D shape model obtained from inversion of delay-Doppler images to strongly constrain Castalia's spin state to one of two possible orientations.

Millimeter Redman et al. (1998, T03-011) reported on high-quality photometry obtained with the JCMT at millimeter and submillimeter wavelengths for asteroids 1 Ceres, 4 Vesta, 6 Hebe, 7 Iris, 16 Psyche, 18 Melpomene, and 216 Kleopatra. They defined an "effective emissivity" and demonstrated that it is a useful means to present the SED of an asteroid over the whole range of wavelengths for which thermal emission dominates the observable flux density. They also investigated the most important physical properties that distinguish the SEDs of asteroids and found, for example, that the SEDs of the M-class asteroids Psyche and Kleopatra have a distinctive shape, with a steep decrease from infrared to radio wavelengths. For these asteroids the effective emissivities at wavelengths near $1 \mathrm{~mm}$ are too low to correspond to physical temperatures in the asteroids' surfaces but are consistent with the presence of large metal fractions in their surface minerals, which would make their surfaces reflective rather than emissive at long wavelengths.

Radiometry No new radiometric observations of main-belt asteroids were published during this reporting period. Instead, published studies involved developing new thermal models, methods for updating previously published radiometric diameters and albedos, and improving the radiometric calibration.

Lagerros, in a series of papers, (1996, T03-001; 1997, T03-003, and 1998, T03-008) investigated the polarization of thermal microwave emission, developed his thermophysical model, which allows for (almost) arbitrary shapes, albedo variegations, beaming, and heat conduction, and presented a refined treatment of thermal infrared beaming.

Mueller and Lagerros (1998, T03-009) studied the use of asteroids as far-infrared photometric standards for ISOPHOT. They applied Lagerros' new thermophysical model, 
utilizing spin-vector solutions, direct size measurements, and the HG-magnitude system, to the observations to derive the thermal properties of selected asteroids. In this way they defined new photometric standards for the far-IR. The absolute accuracy for thermal flux or lightcurve predictions is $5 \%$ to $10 \%$ for the best-observed objects and $10 \%$ to $20 \%$ for objects with lower-quality data. The methods and procedures presented in this paper were included in the first update of the ISOPHOT calibration in 1998. Mueller (1998, T03-015) further discussed the prospects for using asteroids as far-infrared photometric standards.

Cohen et al. (1998, T03-010) described their efforts to seek "closure" in their infrared absolute calibration scheme by comparing 5 through 14 micron airborne spectroscopy of the asteroids Ceres, Vesta, and Pallas, absolutely calibrated through reference stars, with "standard thermal models" and "thermophysical models" for these bodies. They concluded that the best match to their observed spectra of Ceres and Vesta is a standard thermal model using a beaming factor of unity. They also report the presence of three emissivity features in Ceres that may complicate the traditional model extrapolation to the far-infrared from contemporaneous ground-based N-band photometry, e.g, that used to support the ISOPHOT. While identification of specific materials that cause these features is not made, they discuss families of minerals that may be responsible.

Whitteborn et al. (1999, T03-017) discuss a comparison of $\alpha$ Bootis and 1 Ceres with an absolute laboratory flux standard at a spectral resolving power of 100 to 200 . The spectrum of the K1.5 III star $\alpha$ Boo was measured from 3 to 30 microns, and that of 1 Ceres from 5 to 30 microns. They argue that while: "these 'standard' spectra do not have the apparent precision of those based on calculated models, they do not require the assumptions involved in theoretical models of stars and asteroids. Specifically, they provide a model-independent means of calibrating celestial flux in the spectral range from 12 to 30 microns, where accurate absolute photometry is not available. The agreement found between the spectral shapes of $\alpha$ Boo and Ceres based on laboratory standards and those based on observed ratios to $\alpha \mathrm{CMa}$ (Sirius) and $\alpha \mathrm{Lyr}$ (Vega), flux-calibrated by theoretical modeling of these hot stars, strengthens our confidence in the applicability of the stellar models as primary irradiance standards."

Harris (1998, T09-022) noting that near-Earth asteroids are relatively small and therefore expected to have less dusty, "rockier", surfaces with higher thermal inertias than mainbelt asteroids, tend to have irregular shapes, and are often observed at large solar phase angles, compared to main-belt objects, reasons that the applicability of the commonly used asteroid thermal models to these objects is not self-evident. Thus, he compared radiometric data in the 4 through 20 micron range for several near-Earth asteroids with model predictions of the thermal emission. He found that for all the objects considered, both the "standard" and "fast rotating" thermal models produce poor fits to the observational data. He describes simple modifications to the standard thermal model that lead to significantly improved fits to the data and uses this to derive revised estimates of the albedos and sizes for a number of near-Earth asteroids and compares them with existing data from other sources. He proposes that the new model be adopted as a default simple thermal model for estimating albedos and diameters of near-Earth asteroids.

Harris and Harris (1997, T03-004) present a simple, approximate method for the recalculation of asteroid diameters and geometric albedos given new, improved absolute visual magnitudes. This method avoids recourse to detailed models of an object's reflected and thermally emitted flux components. They demonstrate the usefulness of the method for revising the diameters and albedos of asteroids by applying it to a number of IMPS asteroids for which recently updated absolute magnitudes are available.

Polarimetry Lupishko and Vasilev (1997, T03-007) describe a polarimetric asteroid database containing all available results on polarimetry of asteroids, including unpublished data obtained at the Sanglok Observatory (Tajikistan) and the Crimean Astrophysical Observatory (Ukraine). The database includes results of polarimetric measurements of 145 asteroids observed for about 400 nights in one or more of the UBVRI bands. The database also in- 
cludes available asteroid polarimetric phase curve parameters and polarimetric albedos for 127 asteroids.

Lupishko (1998, T03-012) presents a comparison of the IMPS albedos with other published geometric albedos of asteroids (ground-based radiometric, polarimetric, and stellaroccultation) and demonstrates that, if the former $V(1,0)$ absolute magnitude system is used instead of the $H, G$ absolute magnitude system (used to derive the IMPS diameters and albedos) then: "(a) systematic errors in the IRAS albedos grow directly proportional to the value of the albedo itself and (b) the polarimetric albedos are intermediate between the IRAS albedos and the ground-based radiometric ones and correspond most closely to the most accurate, stellar-occultation albedos."

Shevchenko and Lupishko (1998, T-03-013) review studies of the optical properties of asteroids obtained from analysis of photometric observations. The mean values of the albedos, color indices, and the phase-angle dependence of the brightness and color are summarized for asteroids of the common taxonomic classes. They discuss the functions used for the approximation of the phase dependences of asteroid brightness and the functions applied for modeling the distribution of brightness over the asteroid disk.

Cellino et al. (1999, T03-018) presented preliminary results of an ongoing observational campaign aimed at deriving albedo estimates for a sample of small asteroids (smaller than $50 \mathrm{~km}$ ) whose albedo was measured by means of IRAS observations. The results obtained so far indicate an overall satisfactory agreement between polarimetric and IRAS albedos, although some small systematic difference (IRAS albedos being on the average slightly larger) cannot be ruled out based on the available data.

Occultation Erikson et al. (1999, T03-006) used photometric observations together with data from an occultation event during the 1995-1996 apparition of asteroid 85 Io to derive its pole orientation, size and shape. The resultant value for the diameter $(164 \mathrm{~km})$ is about $5 \%$ larger than the IMPS diameter.

\subsection{Binary and family studies}

Major advances have been done in recent years in the field of physical studies of asteroid families. This includes both observational studies, mainly using spectroscopic techniques (Di Martino et al. 1997, T04-004; Zappalá et al. 1997, T04-006; Doressoundiram et al. 1998, T04-009; Florczac et al. 1998, T04-013) and theoretical investigations aimed at deriving from present physical data some insight on the original events which were responsible of family formation. In particular, Zappalá et al. (1996, T04-002) developed a method for reconstructing the original ejection velocity fields in family-forming events. Marzari et al. (1996, T04-001) and Asphaug (1997, T04-007) proposed models of the Vesta family and its possible history, while Davis et al. (1999, T04-012) discussed the fact that the lack of an observable family associated with the large M-type asteroid 16 Psyche raises puzzling questions about the present knowledge of the overall process of collisional evolution of the main belt, and on the currently accepted interpretation of M-type asteroids as metal-rich objects. Doressoundiram et al. (1997, T04-008) discussed the hypothesis that collisional events producing families should likely produce also relevant numbers of binary systems among family members. Binary systems have been another major field of research recently, mainly following the discoveries of the Ida-Dactyl system and the radar observations of possible binaries in the NEA population. Giblin et al. (1998) have analyzed impact ejecta rotational bursting as a possible mechanism for producing stable Ida-Dactyl systems. Scheeres et al. (1998, T04-011) in turn, analyzed the dynamics of orbits close to the asteroid 4179 Toutatis. Pravec and Hahn (1997, T04-005) and Pravec et al. (1998, T04-014) looked for some cases in which photometric properties suggest a possible binary nature for some objects, and Bottke and Melosh (1996, T04-003) suggested that binary asteroids could explain the presence of doublet craters observed on terrestrial planets. 


\subsection{Spectra, taxonomies, and compositions}

Spectra of asteroids are important for studies of surface compositions and is a growing field of asteroid research. The question if low-albedo asteroids are thermally metamorphosed was studied by Vilas and Sykes (1996, T05-002), and Chapman (1996, T05-003) discussed the effects of space weathering on S-type asteroids. Merenyi et al. (1997, T05-014) developed a tool for prediction of water by observing features shortwards of 3 microns. Barucci et al. (1998, T05-018) made a spectroscopic survey of C-type asteroids in order to study features indicating aqueous alteration on the surfaces.

Dumas et al. (1998, T05-019) made I-J-H-K band spectroscopy of low-albedo objects in the outer Solar system. Lucy and Keil (1998, T05-022) studied the influence of temperature on the spectra of A-type asteroids and the spectral features of two M-type asteroids were discussed by Busarev (1998, T05-017).

A survey of the compositional types of the Hilda asteroids were made by Dahlgren et al. (1997, T05-006) and Florczak et al. (1999, T05-023) observed the Themis family.

Studies devoted to individual asteroids such as 4 Vesta, 6 Hebe and 253 Mathilde were made by Gaffey (1997, T05-012), Cochran and Vilas (1998, T05-020), Migliorini et al. (1997, T05-013) and Doressoundiram et al. (1997, T05-007).

\subsection{Origins, impacts, orbital and collisional evolution}

A great amount of work has been devoted to topics related to the origin and collisional evolution of asteroids, including theoretical and experimental studies of the physics of catastrophic break-up phenomena. The early phases of the asteroid belt history have been investigated by Ida and Lin (1996, T06-001), who studied the influence of long-term gas drag effects in the Solar Nebula, while Cyr et al. (1998, T06-025) analyzed the distribution of water ice in the early nebula, which should be related to the present radial trend in the water of hydration bands in present asteroid spectra. From a review of presently published data, Meibom and Clark (1999, T06-030) concluded that ordinary chondritic material has never been abundant in the asteroid belt, which should have been dominated mostly by carbonaceous material. The effect of cometary bombardment of the primitive asteroid belt has been also analyzed by Brunini and Gil-Hutton (1998, T06-029). More in general, the overall collisional evolution of the asteroid population, both in the main belt and in the Trojan clouds, has been the subject of a large number of papers. A brief list includes Marzari et al. (1997, T06-008), Reach et al. (1997, T06-012), Durda and Dermott (1997, T06-014), Dahlgren (1998, T06-019), and Gil-Hutton and Brunini (1999, T06-032). In particular, refined methods for the computation of collisional probabilities and distributions of impact velocities have been published by Vedder (1996, T06-003), Manley et al. (1998, T06-020), and Dell'Oro and Paolicchi (1998, T06-027). As for impact studies, much advancement has been obtained from the theoretical point of view (Love and Ahrens 1996, T06-004; Nolan et al. 1996, T06-005; Bottke et al. 1997, T06-010; Melosh and Ryan 1997, T06-013; Hartmann and Gaskel 1997, T06-015; Keil et al. 1997, T06-017; and Ryan and Melosh 1998, T06-022). As for experiments, new results have been published by Giblin and Farinella (1997, T06-011), Horz and Cintala (1997, T06-016), and Giblin et al. (1998, T06-023), while HST observations of the impact excavation in the asteroid 4 Vesta have been published by Thomas et al. (1997, T06-018).

\subsection{Asteroids visited (or to be visited) by spacecraft}

Four papers based on spacecraft flyby data were published during the reporting period and an additional twelve either on asteroid flyby targets prior to the actual flyby or involving asteroids with future scheduled (or proposed) flybys.

253 Mathilde: Basilevsky (1997, T07-003) discussed images, Yeomans et al. (1997, T07014) presented an early estimate of the mass, and Veverka et al. (1997, T07-015) presented and discussed early images of this C-class NEAR asteroid flyby target. 
951 Gaspra: Menichella (1997, T07-008) tested the hypothesis that a single, very energetic breakup event which locally injected debris into the main-belt population with a size distribution unrelated to the equilibrium value is responsible for the observed cumulative distribution of craters as a function of diameter on the surface of Gaspra, a distribution definitely steeper than the theoretically derived value expected for a projectile population in collisional equilibrium. His results show that this hypothesis is unlikely in the case of Gaspra because: "... it requires two ad hoc assumptions: (1) that the assumed impact should have involved two relatively large asteroids, hence it's a priori probability of occurring during the whole history of the Solar System would have been low; (2) and that this event should have occurred as recently as $10 \mathrm{Myr}$ ago."

Cheng et al. (1997, T07-006) and Cheng (1997, T07-009) present an overview of the NEAR mission. Acuna et al. (1997, T07-007), Cole et al. (1997, T07-013), Goldsten et al. (1997, T07-012), Hawkins et al. (1997, T07-010), Vilas et al. (1997, T07-005), and Warren et al. (1997, T07-011) discuss various details of the NEAR mission.

Michel et al. (1996, T07-001) describe the dynamical evolution of two near-Earth asteroids to be explored by spacecraft: 433 Eros and 4660 Nereus. Rossi and Fulchignoni (1999, T07-002) using the limited data presently available on the ROSETTA spacecraft flyby asteroids (currently 3840 Ministrobel (S-class), 2703 Siwa, and 140 (C-class) calculated, among other things, the orbital stability zone, defined as the union of all the numerically integrated orbits showing long-term stability, for each of the target asteroids. They concluded that the particular shapes of these zones suggest it would be wise to have the spacecraft's close approach out of the orbital plane of the asteroids. Barucci et al. (1998, T07-016) noting that the final selection of the two Rosetta target asteroids will be made in a successive phase of the Rosetta project development, when the engineering parameters will be frozen, present spectroscopic observations of the possible Rosetta candidates and suggest including asteroid 140 Siwa due to its "primitive" compositional classification.

\subsection{The asteroid-meteorite connection}

A more complete derivation of the Yarkovsky effect (Farinella et al. 1998, T08-038; Morbidelli and Brett 1998, T08-045; Vokrouhlicky 1999, T08-061) has shown that the delivery of material from the asteroid belt to Earth may be much quicker than previously believed, strengthening our belief that meteorites are indeed representative samples of the asteroid belt.

Spectra and physical arguments (Migliorini et al. 1997, T08-066; Gaffey et al. 1998, T08-050) have suggested that the large asteroid 6 Hebe is the source of both H-class ordinary chondrites and Type III iron meteorites, a significant fraction of all meteorites in our collections today. Key to the arguments has been the role of space weathering, the possibility that surface processes could weaken and redden the strong mineral bands seen in ordinary chondrite material, bringing them closer to observed asteroid spectra. To this end, laboratory work attempted to reproduce the heating effect of impacts (Moroz et al. 1996, T08-003) and the reduction of surface material by solar wind interactions (Dukes et al. 1999, T08-055) has produced trends in spectra similar to this space weathering.

Dark asteroids and carbonaceous meteorites Moroz et al. (1998, T12-008) noted the similarities in spectral features, and possibly in chemistry, between dark asteroids and terrestrial solid bitumens. Kimura and Ikeda (1998, T08-047) and Krot et al. (1999, T08-056) have developed a detailed understanding of the aqueous alteration of CV carbonaceous meteorites; Sato et al. (1997, T08-022), Shestopalov et al. (1998, T08-031), and Fornasier et al. (1999, T08-054) mapped out the infrared spectra of these hydrated carbonaceous meteorites with an eye to telescopic observations of dark asteroids. Sato et al. (1997, T08-022) found a good match between the three-micron band of the CR meteorite Renazzo and the B-type asteroid 2 Pallas, but a poorer fit to the G-type asteroid 1 Ceres. Burbine (1998, T08-042) also notes that Ceres is spectrally different from known meteorites in the 3 micron region; and although two other G-type asteroids, 19 Fortuna and 13 Egeria, have optical spectral 
properties that are consistent with $\mathrm{CM}$ chondrites, Egeria at least does not match as well in the near-infrared.

Meteorites and asteroid structure Detailed modelling of asteroid collision and re-accretion (Ryan and Melosh 1997, T06-013; Asphaug 1997, T12-005), asteroid shapes (Richardson et al. 1998, T09-025), and observations of meteorite densities (Consolmagno et al. 1998, T09-049) compared to asteroid densities (Veverka et al. 1997, T07-015; Mitchell et al. 1996; Hilton 1997, T01-004; 1999, T01-011; but see Dahlgren et al. 1998, T06-019, for a possible exception to this trend) all suggest that asteroids have a rubble-pile structure. Contrasting meteorite and asteroid densities suggests that $S$ asteroids observed to date are $10-30 \%$ void space (macroporosity, voids on a scale of centimeters to meters) while Mathilde may have as much as $50 \%$ macroporosity. Wilson et al. (1999, T01-013) produced such macroporous rubble pile asteroids in their computer modelling of asteroid reaccretion.

Iron meteorites and mesosiderites show evidence of fragmentation and re-accretion (Haack et al. 1996a,b; T08-001, T08-002). Stony meteorites also appear to have experienced multiple impact events (Kunz et al. 1997, T08-023) and the partial melting of meteoritic material (Yolcubal et al. 1997, T08-016).

Shirey and Walker (1998, T08-030) noted that magmatic iron meteorites indicate asteroidal core crystallization within the first 10-40 million years of Solar System evolution; Horan et al. (1998, T08-035) determined from W-182 isotopic systematics that all of the iron meteorite parent bodies underwent metal segregation within $5 \mathrm{Ma}$ of each other, apparently independently of their composition, size, and time of metal crystallization. This implies that the high temperatures necessary for core segregation were achieved at essentially the same time in a variety of parent bodies of varying size and composition.

Thermal alterations are seen in the petrologic evolution of CM (Zolensky et al. 1997, T08-014) and CV (Krot et al. 1998, T08-046) carbonaceous meteorites; the chemical zoning in pallasites (Miyamoto 1997, T08-017); and the effects of immiscibility of phases on iron meteorites (Ulff-Moeller 1998, T08-041). Bennet and McSween (1996, T08-009) and Akridge et al. (1998, T08-037) modelled the thermal evolution of asteroids and stony meteorites; Chabot and Drake (1999, T08-058) modelled iron meteorite formation in asteroid cores.

The HED Parent Body (Vesta): Ghosh and McSween (1998, T12-007) developed a detailed thermal model for Vesta, thought by many to be the Howardite-Eucrite-Diogenite (HED) meteorite parent body. Righter and Drake (1996, T08-005; 1997, T08-027) presented views of the HED parent body differentiation history. Models by Warren (1997, T08-028) and Treimann (1997, T08-021) put constraints on its geochemical evolution; Yamaguchi et al. (1996, T08-004) discussed its crustal metamorphism, and Wilson and Keil (1997, T08024) the fate of pyroclasts on its surface. Thermal models by Haack et al. (1996b, T08-002) indicated that the mesosiderites (a mixture of metal and basaltic material reminiscent of HED meteorites) probably cooled in an asteroid some $200-400 \mathrm{~km}$ in radius, consistent with slow cooling at depth $<1 \mathrm{My}$ after metal and silicate were mixed around 4.4 Gy ago.

Once formed, the surface of the HED parent suffered repeated impacts to send material eventually on Earth-crossing orbits (Asphaug 1997, T04-007). Different breccia clasts in the howardite Kapoeta (Pun et al. 1998, T08-044) illustrate the repetitive nature of impact comminution and lithification in the regolith of the HED parent body. Welten et al. (1997, T08-026) concluded from the cosmic ray exposure ages of diogenites that two major impact events $22 \mathrm{Ma}$ and $39 \mathrm{Ma}$ ago could explain more than half of all HED exposure ages, but needed at least five impact events to explain all ages $<50 \mathrm{Ma}$.

Most models for HED and iron meteorite formation involve a parent body with a large iron core and olivine-rich mantle, but there are no such olivine meteorites in our collections. Burbine et al. (1996, T08-007) suggested that all differentiated parent bodies (except Vesta) had their crusts and mantles stripped very early in the age of the solar system. The resulting basaltic and olivine-dominated metal-free fragments could have been completely comminuted by subsequent collisions; only the stronger iron-rich meteorites would remain as the final tracers of this differentiation history. 


\subsection{Near-Earth asteroids}

NEOs, their origin and evolution, and their relationship with impact hazards on Earth have been one of the most important fields of activity in the past three years. From the observational point of view, a great effort has been devoted to observing strategies, discovery, follow-up, and physical characterization of these objects. A list of published papers includes Lazzarin et al. (1997, T09-005), Pravec et al. (1997a,b, T09-007, T09-010), Prokof'eva et al. (1997, T09-018), Mukai et al. (1997, T09-058), Spahr et al. (1997, T09009), Ishiguro et al. (1997, T09-017), Boattini and Carusi (1997, T09-019), Davies et al. (1998, T09-023), Hicks et al. (1998, T09-024), Rabinowitz (1998, T09-027), Harris et al. (1998, T09-028), Whiteley and Tholen (1998, T09-031), Lupishko and Di Martino (1998, T09-036), Harris (1998, T09-039), Muinonen (1998, T09-040).

NEO radar observations have been very successful, as shown by Benner et al. (1997, T09-048; 1999, T09-051), Hudson and Ostro (1998, T09-029), and Ostro et al. (1999, T09047). Also, some refinements in the models aimed at describing the thermal IR emission from NEOs have been made (Harris 1998, T09-022; cf., Sec. 2.3). The origin and continuous supply of NEOs has been analyzed by different authors, including Bottke et al. (1996, T09-003), Harris and Bailey (1996, T09-004), Rabinowitz (1997a,b; T09-008, T09-011), Rubincam (1998, T09-032), Greenberg (1998, T09-045). In particular, the role of dynamical families as possible sources of NEOs has been discussed by Migliorini et al. (1997, T09-015), Gladman et al. (1997, T09-059), and Zappalá et al. (1998, T09-026), while the dynamical evolution of bodies in NEO orbits has been analyzed by Michel (1998, T09-043). Moreover, Richardson et al. (1998, T09-025) have analyzed the effects of tidal distortion in case of possible close encounters with the Earth.

In one of the most important new result related to the problem of NEA origin, Migliorini et al. (1998, T09-060) discuss the fundamental role of the Mars-crossing asteroids as a possible source of $\mathrm{km}$-sized NEAs. They used orbital dynamic simulations to demonstrate that many main belt asteroids are driven toward Mars-crossing orbits by numerous weak mean motion resonances, which slowly increase their eccentricity. They showed that about half the Mars-crossing asteroids become Earth-crossing asteroids in less than 20 million years. This scenario quantitatively explains the observed number of large Earth- and Marscrossing asteroids.

A very popular topic has been, as noted above, the evaluation of the frequency of close encounters with the Earth and the related impact hazard. Papers on this subject have been published by Koeberl (1996, T09-001), Carusi and Dotto (1996, T09-003), Yabushita (1997, T09-006), Steel (1997, T09-012; 1998, T09-042), Sitarsky (1998, T09-020), Hills and Goda (1998, T09-038), Vogel (1998, T09-044).

Particular attention has been devoted to new analysis of the 1908 Tunguska event (Trayner 1997, T09-013; Sekanina 1998, T09-037; Foschini 1999, T09-046). Evidence for a late Triassic multiple event has been presented by Spray et al. (1998, T09-035), while the existence of possible artificial biases in searches aimed at identifying periodicities in terrestrial impact cratering rates has been discussed by Jetsu (1997, T09-050). Finally, Yabushita (1998a,b; T09-033, T09-034) has quantitatively discussed the problem of deflecting objects on a collision course with the Earth.

\subsection{Trojan asteroids of Mars, Jupiter, et al.}

The techniques of orbital and collisional mechanics developed for understanding the evolution of the asteroids in the main belt have also been applied to Jupiter Trojan asteroids. Giorgilli and Skokos (1997, T10-001) showed that a region of Jupiter Trojan Lagrangian space at least big enough to include a few known asteroids is stable over the age of the Universe, while Dell'Oro et al. (1998, T10-002) have included Jupiter perturbations in the Trojan region to calculate collision probabilities within the known population of Jupiter Trojans. 
Tabachnik and Evans (1999, T10-003) used the orbits of the two known and two suspected Mars Trojan asteroids to investigate the stability of Martian Trojan orbits. They found that stable orbits occupy a band of inclinations between $15 \mathrm{deg}$ and $40 \mathrm{deg}$ and longitudes between $240 \mathrm{deg}$ and $330 \mathrm{deg}$ at the L5 Lagrangian point. In the vicinity of the L4 point, stable Mars Trojans inhabit two bands of inclinations (15 deg $<i<30 \mathrm{deg}$ and $32 \mathrm{deg}<i<40 \mathrm{deg}$ ) with longitudes restricted between $25 \mathrm{deg}$ and $120 \mathrm{deg}$. Both confirmed Martian Trojans lie deep within one of the stable zones, which, the authors conclude, suggests that they may be of primordial origin. The two potential Martian Trojans are not presently in Trojan orbits but will enter the sphere of influence of Mars within half a million years. They state that the number of undiscovered primordial objects with sizes greater than $1 \mathrm{~km}$ may be as high as 50 .

\subsection{Distant asteroids: Centaurs and Trans-Neptunian objects}

Centaurs Jedicke and Herron (1997, T11-004) determined the efficiency of the Spacewatch system (which has discovered three new Centaurs and made a serendipitous detection of 2060 Chiron) as a function of an object's apparent visual magnitude and rate of motion. Convolving these efficiencies with the scanning pattern of the telescope and a theoretical orbital distribution for the Centaurs allowed them to determine the system's efficiency as a function of their absolute magnitude. The efficiency is nonzero for objects larger than about $40 \mathrm{~km}$ diameter and has a maximum of about $3.4 \%$ for Centaurs with diameters around $600 \mathrm{~km}$. They conclude that at the $99 \%$ confidence level, for the orbital distribution used in their analysis and in the absolute magnitude range $-4<H<10.5$, there must be fewer than $\sim 2000$ Centaurs. This implies that the Centaur population may be as great or larger than the set of main belt asteroids in the same size range. They estimate that there may be a total of only three objects of Chiron's size (about $200 \mathrm{~km}$ diameter) or larger.

Weintraub et al. (1997, T12-005) obtained visible and near-infrared broadband photometry of the Centaur objects 1995 GO and 5145 Pholus. They confirmed the extremely red V-J color and nearly solar J-H and H-K colors of 5145 Pholus. Their V-J data reveal that $1995 \mathrm{GO}$ is as red as the reddest D-class asteroid, 1748 Mauderli, but not nearly as red as 5145 Pholus. They suggest that the red color of $1995 \mathrm{GO}$ is due to the presence of organic-rich materials produced by particle bombardment and ultraviolet irradiation of surface grains, a process that may be widespread among the Centaur and trans-Neptunian object populations. They demonstrate that the V-J data for the Centaurs suggests that V-J increases with increasing semimajor axis and conclude that, if this correlation is correct, then the red colors of the Centaurs 5145 Pholus and 1993 HA2 may be typical of fairly pristine Centaur objects. In contrast, the progressively less red colors of $1995 \mathrm{GO}$ and 2060 Chiron may be the result of an increased rate of resurfacing of these objects as they migrate progressively closer to the Sun.

Barucci et al. (1999, T11-015) performed 378 to 750-nm spectroscopic observations of five Centaurs. Their results show a great diversity among the reflectances; the colors in this spectral region do not seem to be related to the perihelion distance of the objects. They also looked for weak cometary emission features, in particular the CN-band emission at 388 $\mathrm{nm}$, but detected no emission features attributable to $\mathrm{CN}$.

Jewitt and Kalas (1998, T11-011) combined new measurements of the thermal emission from 1997 CU26 with published optical photometry to determine the geometric albedo $(0.045 \pm 0.010)$ and effective diameter $(302 \pm 30 \mathrm{~km})$. They concluded that, while these values are model dependent, they clearly show that 1997 CU26 is the largest of the known Centaurs and that its surface is very dark. Brown and Koresko (1998, T11-012) report the detection of the 1.5 and 2.0 micron absorption bands due to water ice in the nearinfrared reflection spectrum of 1997 CU26. The water ice bands are weaker than those detected on the surface of any other solar system body; the spectrum is well fit with a model surface consisting predominantly of a neutral dark absorbing substance with only $\sim 3 \%$ areal coverage of water ice. The spectrum thus appears very different from that of the Centaur 5140 Pholus, although both objects are of similar brightness and are at similar 
heliocentric distances. See also Brown et al. (1998, T11-013) for a report on the detection of water ice on Neptune's distant irregular satellite Nereid.

Trans-Neptunian objects Jewitt et al. (1998, T11-008) describe a large-area ecliptic survey designed to assess the sky-plane surface density of bright Edgeworth-Kuiper belt objects (trans-Neptunian objects, TNOs). They used an $8192 \times 8192$ pixel CCD mosaic to image $51.5 \mathrm{deg}^{2}$ to a $50 \%$ detection threshold magnitude of $R=22.5$. Thirteen new TNOs were identified in the survey, including some of the brightest and, presumably, largest known examples. They used Monte Carlo models to assess the effects of observational bias and to examine (1) the size distribution of bright objects in the Edgeworth-Kuiper belt, (2) the possible existence of a cutoff in the size distribution at large radii, (3) the intrinsic ratio of Plutinos to nonresonant ("classical") Edgeworth-Kuiper belt objects, (4) the intrinsic ratio of populations in the 3:2 and 2:1 mean motion resonances, and (5) the radial extent of the Egdeworth-Kuiper Belt.

Gladman et al. (1998, T11-009) conducted pencil-beam searches for outer solar system objects to an $R$ limiting magnitude of about 26 . The fields were searched using software recombinations of many short exposures shifted at different angular rates in order to detect objects at differing heliocentric distances. Five new trans-Neptunian objects were detected in these searches. They estimate there are about 90 trans-Neptunian objects $/ \mathrm{deg}^{2}$ brighter than approximately 25.9. They note that this estimate is a factor of 3 above the expected number of objects based on an extrapolation of previous surveys with brighter limits and appears consistent with the hypothesis of a single power-law luminosity function for the entire trans-Neptunian region. Maximum-likelihood fits to all self-consistent published surveys with published efficiency functions predicts a cumulative sky density $\Sigma(<R)$ obeying $\log \Sigma=0.76(\mathrm{R}-23.4)$ objects $/ \mathrm{deg}^{2}$ brighter than a given magnitude $\mathrm{R}$.

Davis and Farinella (1997, T11-002) studied the collisional evolution of EdgeworthKuiper belt objects and concluded that collisional evolution is substantial in the inner part, but its intensity decreases with increasing distance from the Sun. In the inner belt, objects with diameters greater than 50 to $100 \mathrm{~km}$ are not depleted by disruptive collisions; hence they reflect the original (formative) population (many of them, however, may have been converted into "rubble piles"). On the other hand, smaller objects are mostly multigenerational fragments, although the original population must have contained a significant number of bodies down to at least a few tens of kilometers in size in order to initiate a collisional cascade. They discussed the delivery of material to the inner solar system and find that most comets coming from the Edgeworth-Kuiper belt would be fragments from larger parent bodies, rather than primitive planetesimals. However, they note that this does not apply to Chiron-sized (diameters greater than $100 \mathrm{~km}$ ) objects, which must be primordial and delivered to the outer Solar System by either dynamical processes or nondisruptive collisions.

Lu and Jewitt (1996, T11-001) observed 4 Centaurs and 14 TNOs and reported a wide dispersion in their BVRI colors that they attribute to the expected steady reddening due to cosmic ray bombardment. They present a model in which the dispersion is produced by stochastic collisional resurfacing and note that, while non-unique, this model provides a plausible match to the observed color distribution.

Davies et al. (1997, T11-003) present visual photometry for TNO 1993 SC that indicates a lightcurve amplitude of less than $0.2 \mathrm{mag}$ and note that this differs from the $0.5 \mathrm{mag}$ amplitude reported by I. P. Williams et al. (Icarus 116, 180-185, 1995). They also obtained VRIJ colors which confirm that $1993 \mathrm{SC}$ has visual and infrared colors closer to Centaur 1993 HA2 than to the extremely red 5145 Pholus.

Green et al. (1997, T11-006) present BVRI photometry of five TNOs and one Centaur and Palshin and Tsygan (1998, T11-014) published BVRI photometry and position measurements for three TNOs. Green et al. report no compelling evidence for a correlation of colors with orbital zones, and consequently no clear mechanism to explain this diversity in terms of the irradiation mantle model. 
Jewitt and Lu (1998, T11-007) measured the optical and infrared colors of five Edgeworth-Kuiper belt objects to search for evidence of spectral diversity among these bodies. They found extreme differences, especially in the measured V-J color indices, that indicate a wide range of surface compositions in the Edgeworth-Kuiper belt. The Edgeworth-Kuiper belt observations show a correlation between the V-J color index and the absolute red magnitude that is significant at the 3 sigma (99.7\%) confidence level. This correlation may indicate that the surface compositions of TNOs are related to their diameters, possibly as a result of the preferential retention of frost by large objects or of the size-dependent effects of collisional resurfacing. However, they note that the current sample is small, and caution that a larger sample must be obtained before the observed correlation can be considered secure.

Magnusson et al. (1998, T11-010) obtained VRI photometry of one Centaur and seven TNOs. They found one object, 1994 JQ1, may be as red as 5145 Pholus, the reddest minor planet previously known. The Centaur 1995 DW2 has more moderate color indices, similar to main-belt asteroids. They reported no light variation above the expected noise was evident, apart from a few outliers. Their four nights of observations with the ESO New Technology Telescope covered 0.52 square degrees during which two previously unknown objects, 1995 FB21 and 1995 GY7, were discovered. They estimate the density of TNOs brighter than $R=24$ to be 5.3 per square degree near the ecliptic.

\subsection{Miscellaneous asteroid-related publications}

Cometary material on Earth? Campins and Swindle (1998, T08-048) examined what a meteorite from a comet might look like, and concluded that though no particular class of meteorite is an obvious candidate, some xenoliths in ordinary chondrite regolith breccias meet many of their criteria for a cometary origin. Mautner et al. (1997, T12-003) examined the implications of a significant infall of cometary material on the biospheres of planets. Feeding meteorite-derived nutrients to terrestrial organisms, they found that solutions of these nutrients in meteorites could provide efficient concentrated biogenic environments, including high phosphate levels (probably the limiting nutrient). But can such material survive entry through the Earth's or another planet's atmosphere? Experiments by Basiuk and Navarro-Gonzalez (1998, T12-009) showed that some simple amino acids, purines, and pyrimidines do not completely decompose even under volatilization at $500^{\circ} \mathrm{C}$ in a nitrogen atmosphere at normal pressure, with the percentage of survival of the order of 1$10 \%$ and biomolecules in dust particles could rapidly sublime at lower temperatures during the atmospheric passage, dissipating in the upper atmosphere without being destroyed.

Active asteroids Even in the absence of cometary volatiles, rocky asteroids could have exospheric activity. Morgan and Killen (1998, T12-011) note that asteroids could have sodium or $\mathrm{OH}$ coronae via the same processes that produce such thin atmospheres on the Moon and Mercury, but such exospheres are probably below present detection limits. Lee (1996, T12-001) suggested that electrostatic levitation of dust on asteroid surfaces could be an effective process of fine particle segregation and transport on asteroids, and might constitute a significant loss mechanism for their smallest particle size fraction during time intervals between large, regolith-dispersing impacts. The process may also contribute to forming coarse-grained near-surface charged "dust spheres" over the sunlit hemispheres of some asteroids.

Asteroid searches Efforts continue to develop systematic, automated surveys to find new asteroids. Spectra alone are not sufficient to identify asteroids; Krisciunas et al. (1998, T12-012) noted that on the basis of Sloan Digital Sky Survey photometry, Cepheids, metalpoor stars, and many types of asteroids are indistinguishable from the stellar locus of field stars. However, Sykes and Moynihan (1996, T12-002) developed general equations for the apparent motions of asteroids, even observed away from opposition, which can be used to distinguish specific asteroid populations. 


\section{THE ASTEROID-COMET INTERFACE}

This section deals with observations and theoretical studies of transition objects with characteristics that render their classification as asteroids or as comets uncertain.

Fernandez et al. (1997) analyzed old POSS images of the transition object 107P/Wilson-Harrington $=$ asteroid 4015 and studied the gas tail. Hicks et al. (1998) presented reflectance spectra of NEOs and extinct comet candidates and found that they have a taxonomic distribution close to that of meteoritic falls. Davies et al. (1998) presented the light curve and colors of $1996 \mathrm{PW}$, an asteroid with the orbital characteristics of a longperiod (LP) comet. They found that it is similar to D-type asteroids. Di Martino et al. (1998) presented spectroscopic observations of three transition objects: (6042) 1990 WW 2, (6144) 1994 EQ 3, 1995 QY 2. The dust-tail of the comet-asteroid 133P/1996 N2 ElstPizarro in 1996 was explained by Boehnhardt et al. (1999) as being due to a short dust emission episode around perihelion passage of the object.

Much theoretical work was done on such objects. Weissman and Levison (1997) speculated about the origin of $1996 \mathrm{PW}$, discussing the possibility that the Oort cloud could also contain some objects from the asteroid belt. Harris and Bailey (1996), Sekanina (1999), and Campins and Swindle (1998) studied the physical evolution from comets to asteroidal objects. Coradini et al. $(1997 \mathrm{a}, \mathrm{b})$ presented results obtained with their nucleus evolution model and tried to establish the conditions under which an active comet nucleus can become dormant or extinct. Harris and Bailey (1998) made a long-term numerical integration of Jupiter-family comets to infer the contribution of cometary asteroids to the NEO population.

\section{COMETS}

Two very bright Comets (C/1995 O1) Hale-Bopp and (C/1996 B2) Hyakutake made their appearance during this triennium (July 1996 through June 1999). While C/Hale-Bopp was observed over very large heliocentric distance ranges starting from more than $7 \mathrm{AU}$ pre-perihelion to even larger distances post-perihelion, $\mathrm{C} /$ Hyakutake made a very close approach to Earth. Thus, one comet provided opportunities to observe coma evolution over large heliocentric distances while the other provided opportunities for detailed studies as a function of cometocentric distances. Cometary X-rays, sodium tails, and many molecules (previously not known to exist in comets) were discovered. Because of space limitations, only a fraction of papers published can be reviewed in this summary.

The DS-1 (New Millennium series) spacecraft was launched October 24, 1998, and Stardust (Discovery series) spacecraft was launched February 7, 1999, to Comet 81P/Wild 2. The Rosetta (ESA Cornerstone series) mission, scheduled for launch January 21, 2003, continues to make excellent progress. The development of ST-4/Champollion (New Millennium series) was canceled and the Discovery series missions Contour and Deep Impact (newly approved) have been put on hold.

\subsection{Nucleus: properties}

Modeling the evolution of thermal and gas flux from a comet nucleus has been improved over the last years. Considering more minor species, better boundary conditions, and multidimensions are important steps to more complete models. Benkhoff and Huebner (1996) calculated the gas flux of $\mathrm{H}_{2} \mathrm{O}$ and minor volatiles of a Jupiter-family comet and showed how the mixing ratios of minor volatiles relative to water vary as a function of heliocentric distance. Benkhoff and Boice (1996) used this model as input to calculate abundances of molecules in the coma. Coradini et al. (1997) studied the evolution of a nucleus taking into account its orbital evolution. They assumed that the nucleus undergoes several close encounters with Giant Planets before becoming a short-period (SP) comet. Benkhoff (1999) discussed the energy balance and the gas flux from the surface of a nucleus. Benkhoff (1999) and De Sanctis et al. (1999) compare active vs. inactive regions on a nucleus. Enzian et al. 
(1997, 1998, and 1999) developed a multi-dimensional model to estimate the temperature and gas production distributions on a nucleus. The spherical model nucleus is assumed to be made up of a porous dust-ice $\left(\mathrm{H}_{2} \mathrm{O}, \mathrm{CO}\right)$ matrix. For Comet $46 \mathrm{P} /$ Wirtanen they confirmed results from one-dimensional calculations, e.g., that the $\mathrm{CO}$ gas release on the surface is quasi-uniformly distributed in contrast to nonuniform water outgassing. Orosei et al. (1999) discussed a numerically improved thermo-chemical evolution model of nuclei. Huebner et al. (1999) compare the status of nucleus models. Rickman and Jorda (1998) studied observational data of Comet $46 \mathrm{P} /$ Wirtanen and found that the nucleus may be in a state of high activity. They suggested that fragmentation of the surface layers may occur near perihelion, leading to additional sublimation from short-lived, icy fragments. There are further papers on structure and evolution of P/Wirtanen by Podolak and Prialnik (1996), Capria et al. (1997), and De Almeida et al. (1996).

There were also large efforts in modeling global, detailed, and local phenomena of comet nuclei. Kührt and Keller (1996) discussed the role of dust within the nucleus and Möhlmann (1996) studied new approaches of cometary activity. Hughes (1996), Greenberg and $\mathrm{Li}$ (1999), and Herique et al. (1999) investigated the interior of a nucleus. Keller and Thomas (1997) discussed the concept of formation of the nucleus of Comet Halley. Due to planned space missions to explore comets, papers focus on target comets such as 46P/Wirtanen and on engineering models. Klinger et al. (1996) reviewed modeling of comet nuclei for engineering studies for space missions to comets. Krolikowska et al. (1999) presented an improved forced-precession model of 46P/Wirtanen. From this model they obtained a range $2.5-10.0$ hours for the spin period. Keller et al. (1999) discussed the relationship between physical bulk properties and porosity.

Unexpected activity of comet nuclei is investigated by several authors. Carbot et al. (1996) studied 29P/Schwassmann-Wachmann 1, Tanigawa et al. (1997) looked into the activity of nucleus fragments of D/Shoemaker-Levy 9, and Prialnik (1997) presented a model for the nucleus of C/Hale-Bopp. Desvoivres et al. (1999) studied the bright condensations observed in the near-nucleus coma of C/1996 B2 Hyakutake. They made the hypothesis that these condensations are induced by fragments of the nucleus. Results are in good agreement with observations if fragment sizes of $20 \mathrm{~m}$ and a density of $300 \mathrm{~kg} / \mathrm{m}^{3}$ are assumed. Banaszkiewicz and Rickman (1996) modeled the evolution of a comet using kinetic theory by coupling physical and chemical models; Markiewicz et al. (1998) studied the evolution of ice surfaces within porous surface layers on comet nuclei. Skorov and Rickman (1998) presented simulations of the gas flow in a Knudsen layer. Skorov (1997) simulated the disequilibrium kinetics of gas in a porous layer. Shoshani et al. (1997) presented a simple model for changing the pore structure and dust grain size distribution in a layer. They calculated the probability for pore blocking and particle trapping when following the evolution of the pore size distribution and the particle size distribution as a function of space and time. Shoshany et al. (1999), used a Monte Carlo model that simulates dust migration in a porous nucleus. Yabushita (1996) and Whipple (1999) investigated modifications of nucleus structure by the transport of super volatiles. Ryabova (1997) modeled the ejection of large dust particles from the nucleus of $1 \mathrm{P} /$ Halley. He proposed a model of a stream of particles ejected from a nucleus into a spacecraft trajectory.

Interesting implications for the formation and physical evolution of Edgeworth-Kuiper belt objects (the potential source of short-period comets) arise from broadband photometry of Centaurs and TNOs. Luu and Jewitt (1996) found a wide dispersion in their optical properties, which they suggest may be due to cosmic ray reddening combined with collisional resurfacing. Barucchi et al. (1999) also find extensive compositional surface variety among Centaurs. Tegler and Romanishin (1998) reported the results of a two-year survey of the broadband optical colors of about one-quarter of the known TNOs. They argued that their colors indicate the presence of two distinct populations: one consisting of objects whose surface colors were only slightly redder than the color of the Sun while the other consisted of the reddest small objects known in the solar system. 
Lifetimes of hypothetical dust particles around large Centaurs and TNOs were investigated by Brown and Luu (1998). They compute dust particle trajectories assuming comet nucleus and solar gravity, and solar radiation pressure. They found particle lifetimes are extremely sensitive to the magnitude of the initial velocity. Yamamoto and Mukai (1998) estimated the release rate of dust grains by the impacts of interstellar dust grains on TNOs. They found that this process is an important source of interplanetary dust particles.

\subsection{Nucleus: origin and evolution}

Comet nuclei are presumed to be the remnants of a large population of icy planetesimals initially in the outer regions of the planetary disk which accreted to form the cores of the giant planets. The breakup of Shoemaker-Levy 9 while in orbit around Jupiter suggests that these objects are nearly strengthless and are most likely 'rubble piles.' The formation of these objects in the solar nebula during the very early epochs of planet formation has been one of the main problems plaguing planetary science. Weidenschilling (1997) has presented the most comprehensive models to date for the formation of comets, which begin with a uniform mixture of microscopic grains in the nebular gas. In these models, particles mainly grow by collisional coagulation since turbulence inhibits gravitational instability. Interestingly, the size distribution of cometesimals develops a narrow peak in the range of tens to hundreds of meters. Thus, these models predict that bodies accreted in this model have low mechanical strength and macroscopic voids in addition to small-scale porosity.

The Oort cloud and long-period comets Fernandez (1997) has reinvestigated the formation of the Oort cloud using Monte Carlo simulations. He found that if the main galactic perturbers were similar to the current ones, the resulting Oort cloud would have probably been too loosely bound to have withstood the disrupting effect of giant molecular clouds over the age of the solar system. The simulation predicts that the combined action of planetary and external forces would have produced a more tightly bound and populous comet reservoir if the solar system formed within a much denser galactic environment, perhaps a molecular cloud and/or an open cluster. However, Bally et al. (1998) has shown the proto-planetary disks in Orion evaporate due to the ultraviolet radiation of the OB stars on timescales less than $10^{5}$ years. This suggests that stars forming in such environments may not form planetary systems that contain gas giants like Jupiter and Saturn. An interesting by-product of Fernandez's scenario of a much denser galactic environment is that we may expect a significant number of planetesimals from the Jupiter and Saturn accretion zones in the Oort cloud.

The most comprehensive model to date of the dynamical evolution of LP comets was presented by Wiegert and Tremaine (1999). They confirmed the conclusion of previous investigators that the observed distribution of LP comet orbits does not match the expected steady-state distribution unless there is fading or some similar physical process that depletes the population of older comets. They can match the observed orbit distribution if the fraction of comets that remains observable after $m$ apparitions is $\propto m^{-0.6 \pm 0.1}$, or if approximately $95 \%$ of comets live for only a few $(\sim 6)$ returns and the remainder last indefinitely. Emel'yanenko and Bailey (1998) estimated the capture probability from nearly parabolic to SP comets to be $\sim 5 \%$.

Matese and Whitmire (1996) reported the detection of a nonrandom distribution in the galactic longitudes of perihelia vectors of LP comets. They show that this is most likely caused by the adiabatic perturbation of the galactic radial tide. The radial component of the galactic tide had been ignored by most investigators.

García-Sánchez et al. (1999) studied Hipparcos proper-motion and parallax data and ground-based radial velocity measurements for nearby stars to find stars that may have passed, or will pass, close enough to the Sun to perturb the Oort cloud. They estimate that one star, Gliese 710 , will have a close approach of $<0.4 \mathrm{pc}$ within $1.4 \mathrm{Myr}$, and that several stars will come within $1 \mathrm{pc}$ during a $10 \mathrm{Myr}$ interval. None of the passing stars perturb 
the Oort cloud sufficiently to create a substantial increase in the LP comet flux at Earth's orbit. Their sample is limited, however, by the incompleteness of the Hipparcos data set.

The Edgeworth-Kuiper belt and Jupiter-family comets The discovery of about 190 (as of June 1999) objects mostly in orbits beyond Neptune showed that this region is richly populated. The objects are divided into Centaurs (inside the Neptune orbit) and EdgeworthKuiper belt objects (trans-Neptunian objects-TNOs). The TNOs have a combined mass of about 0.1 Earth masses (Jewitt et al. 1996). Within the Edgeworth-Kuiper belt are the 'classical' objects, the extended swarm of scattered TNOs (such as 1996 TL66), and the 'Plutinos' (objects in a mean motion resonance orbit). The 'Plutinos' make up about $40 \%$ of the discovered objects in the 30-50 AU region (Jewitt et al. 1998).

Although it is widely accepted that Jupiter-family comets originate in the transNeptunian region, several papers discussed the details of this scenario. Farinella and Davis (1996) argued that collisions are a plausible mechanism for injecting TNOs of 1 to $10 \mathrm{~km}$ in diameter into dynamical resonances, where they can be transported into the inner solar system to become Jupiter-family comets. Ip and Fernandez (1997), discussed the transNeptunian comets, which initially are stored in the $2: 3$ orbital resonance with Neptune. They argued that random gravitational scattering by TNOs with diameters in the $100 \mathrm{~km}$ range could be of potential importance in destabilizing the resonant orbits and hence act as an injection mechanism of SP comets. Duncan and Levison (1997) presented an alternative mechanism: dynamical leakage from an extended disk of comets formed by encounters with Neptune during the early dynamical evolution of the outer solar system. The transfer of bodies from the trans-Neptunian region to the terrestrial zone was simulated by Levison and Duncan (1997). They found excellent agreement between their models and the observed Jupiter family. The relationship between the Edgeworth-Kuiper belt and the origin of Jupiter-family comets was reviewed by Morbidelli (1998). The origin of comet P/Encke was considered by Steel and Asher (1996).

Davis and Farinella (1997), Stern (1996), Stern and Colwell (1997a,b), and Kenyon and Luu (1998) discussed the formation of TNOs by binary accretion in the primordial trans-Neptunian disk. An amount of mass was needed in the primordial Edgeworth-Kuiper belt that far exceeded what is currently observed. Morbidelli and Valsecchi (1997) argued that Earth-mass planetesimals could have depleted most of this primordial mass by (1) injecting most of the bodies from the stable into the unstable regions in the inner belt and (2) enhancing the role of mutual catastrophic collisions in the outer belt.

\subsection{Gas coma and photochemistry}

Two of the brightest comets of the past two decades were observable in this Triennium. Descriptions of the observations of Comets Hyakutake and Hale-Bopp are in Sect. 4.6. Much observational and modeling work on $\mathrm{C} / \mathrm{Halley}$ and other comets also continued. The selection of 46P/Wirtanen as the Rosetta mission target heightened interest in this comet and resulted in a spate of observations to determine the gas abundances (Farnham and Schleicher 1998; Fink et al. 1998; Jockers et al. 1998; Schulz et al. 1998; Stern et al. 1998). The SOHO spacecraft detected the Ly- $\alpha$ coma of this comet (Bertaux et al. 1999).

The distribution of $\mathrm{C}_{2}$ in comet comae has long defied modeling attempts. It has been surmised that its production is a three-step process. Combi and Fink (1997) explored $\mathrm{C} /$ Halley data to show that either a three-step process with isotropic ejection of daughters, or a CHON source, might explain the observations. Feldman et al. (1997) reported UV observations of Comets C/1979 Y1 (Bradfield), 1P/Halley, C/1989 X1 (Austin), and C/1990 K1 (Levy) obtained with IUE and 103P/Hartley 2 and C/1991 T2 (ShoemakerLevy) obtained with $\mathrm{HST}$ concentrating on the $\mathrm{CO}_{2} / \mathrm{CO}$ ratio. All but $\mathrm{C} /$ Levy showed similar $\mathrm{CO}_{2} / \mathrm{H}_{2} \mathrm{O}$; however, the two comets observed with $\mathrm{HST}$ appear deficient in $\mathrm{CO}$ (but not $\mathrm{CO}_{2}$ ). The authors suggested this may imply that the overall level of activity of a comet may be related to its relative CO abundance. Meier and A'Hearn (1997) modeled the SI triplet emissions near $1814 \AA$ in spectra of 19 comets observed with IUE and HST. 
They derive total sulfur abundances relative to water of 0.001 to 0.01 . Sulfur in volatile species (ice) appears to be depleted compared with solar abundances. Tozzi et al. (1998) point out the importance of the metastable $\mathrm{C}\left({ }^{1} \mathrm{D}\right)$ line as a diagnostic of carbon in comet comae. They model IUE observations of the UV line in several comets. Their models suggest that photodissociation of $\mathrm{CO}$ is the primary source of this line. ISO observed Comet $103 \mathrm{P} / \mathrm{Hartley} 2$ and detected emissions of $\mathrm{H}_{2} \mathrm{O}$ and $\mathrm{CO}_{2}$ with $\mathrm{CO}_{2} / \mathrm{H}_{2} \mathrm{O} \sim 8 \%$. This is an unusually large production of $\mathrm{CO}_{2}$ relative to $\mathrm{C} / \mathrm{Halley}$ and other comets (Colangeli et al. 1999). Kawakita et al. (1997) observed Comets Tabur and Liller. They suggest these two comets were once one and split. However, they detect different gas-to-dust ratios in the two comets, implying inhomogeneity in the original nucleus.

Häberli et al. (1997b) have developed a new MHD model for understanding ions in comets, which takes into account the dynamics and chemistry of the ions and the solar wind. This model was applied to observations of $\mathrm{C} / \mathrm{Halley.} \mathrm{A} \mathrm{multifluid-chemical} \mathrm{model}$ was used by Rodgers and Charnley (1998) to investigate the production of HNC and HCN in comets. This model was compared with observations of Comets Hale-Bopp and Hyakutake. Crifo and Rodinov (1997a,b) have modeled the innermost regions of the coma around nonspherical comet nuclei.

\subsection{Dust coma and tail}

Dust coma observations and parameter constraints of Comet $46 \mathrm{P} /$ Wirtanen were presented by Epifani et al. (1999), Fulle (1999), Colangeli et al. (1998), Jockers et al. (1998), Farnham and Schleicher (1998), Lamy et al. (1998b), Schulz et al. (1998), Cartwright et al. (1997), and Fulle et al. (1997c).

Results from dust coma structure analyses, photometry and spectroscopy monitoring of CCD imaging in the visible wavelength range were published for Comets C/1991 T2 Shoemaker-Levy and C/1993 Y1 McNaught-Russel by Waniak et al. (1998), Comet 19P/Borrelly by Lamy et al. (1998a) and Fulle et al. (1997a), 1P/Halley by Schleicher et al. (1998), C/1982 M1 Austin by Rosenbush et al. (1997) and Svoren (1996), C/1996 Q1 Tabur and C/1988 A1 Liller by Kawakita et al. (1997), 26P/Grigg-Skjellerup by McBride et al. (1997), 107P/1949 W1 Wilson-Harrington by Fernandez et al. (1997), C/1980 E1 Bowell, C/1979 Y1 Bradfield, 23P/Brorsen-Metcalf, 21P/Giacobini-Zinner, C/1990 K1 Levy, and 38P/Stephan-Oterma by Sanzovo et al. (1996), and 4P/Faye by Grothues (1996). Similar observations in the IR wavelength range are available from $65 \mathrm{P} / \mathrm{Gunn}$ by Colangeli et al. (1998) and 1P/Halley by Woodward et al. (1996),

Colangeli et al. (1999) measured the color temperature of the dust coma of Comet $103 \mathrm{P} /$ Hartley using ISO. They could not detect the silicate emission at $11 \mu \mathrm{m}$. COBE detections of dust comae and tails of Comets C/1989 Q1 Okazaki-Levy-Rudenko, C/1982 M1 Austin, 73P/Schwassmann-Wachmann 3, and C/1990 K1 Levy were analyzed by Lisse et al. (1998). The $10 \mu \mathrm{m}$ silicate feature was detected by Hanner et al. (1996) in Comets $19 \mathrm{P} /$ Borrelly and $4 \mathrm{P} /$ Faye at $r \approx 1.5 \mathrm{AU}$, but was not found in Comet $24 \mathrm{P} /$ Schaumasse.

Kiselev (1999) argues that filter properties can significantly influence polarimetry measurements of comet dust and therefore photometry and polarimetry should always be done in parallel to obtain accurate results. The composition of comet dust was constrained from spectropolarimetry measurements by Kolokolova and Jockers (1997). Polarimetry observations of Comet 47P/Ashbrook-Jackson were collected by Renard et al. (1996).

Greenberg discusses the possibilities to explain the extended CO source in $1 \mathrm{P} / \mathrm{Halley}$ by gas release from organic core-mantle particles in the coma (1998). The optical color and polarization properties of comet dust is modeled by Kolokolova et al. (1997). The polarization and the $11 \mu \mathrm{m}$ silicate emission feature of aspherical grains was calculated by Yanamandra-Fisher and Hanner (1999) for different dust properties and viewing aspects. The thermal emission spectrum and silicate emission of Comet 19P/Borrelly was calculated by $\mathrm{Li}$ and Greenberg (1998). The orbit dynamics of large dust grains orbiting comet nuclei is described by Fulle (1997). 
Laboratory experiments with amorphous magnesium silicate smoke particles showed remarkable similarities with the 9 and $11 \mu \mathrm{m}$ emission features in comet dust (Hallenbeck et al. 1998). A fit of the same features was attempted by Colangeli et al. (1996) using pyroxene- and olivine-type submicrometer grains.

The dust trails of 22P/Kopff were observed with ISO and analyzed by Davies et al. (1997b). Wide-field images of the dust tail of 1P/Halley taken in 1986 were analyzed by Grothues and Schmidt-Kaler (1996). From a dust tail analysis Fulle et al. (1998) explained the rapid fading of C/1996 Q1 Tabur by a seasonal effect, complete mantling, or exhaustion of the ice reservoirs of the nucleus. A revised fragmentation model for the explanation of striae in dust tails of bright comets was introduced by Nishioka (1998).

\subsection{Plasma}

Solar-wind interaction models The basic physics of the interaction with an active comet near the Earth's orbit was established by measurements from spacecraft encounters and reproduced by modeling. Mass loading of the solar-wind field lines by cometary ions decelerates the supersonic and super-Alfvénic flow. This leads to formation of a bow shock, a pileup region (in density and in magnetic field), a diamagnetic cavity, and a plasma tail. While there is overall understanding, improvements to the physical model and computational technique continue. These include: (1) using a multi-scale approach so that the entire atmosphere is treated while still resolving the shock and the diamagnetic cavity (Gombosi et al. 1996); (2) exploring the need for an additional energy source from supra-thermal electrons (Häberli et al. 1996); (3) establishing a gas release value below which no bow shock forms (Bogdanov et al. 1996); (4) clarifying structure in the inner coma (Sharma and Milikh 1996; Lindgren et al. 1997); (5) introducing a two-fluid (solar wind protons and cometary ions) approach (Gan-Baruch et al. 1998); (6) investigating the importance of charge exchange in the ionization of neutral molecules (Khabibrakhmanov and Summers 1997); and (7) showing that mass-loading shocks are among the most complex in the solar system (Coates et al. 1997). A common theme in some of the model work is the desire to reliably relate observed $\mathrm{H}_{2} \mathrm{O}^{+}$abundances to the neutral gas production rates (Häberli et al. 1997b; Rauer et al. 1997; Wegmann et al. 1999). Note that the ion pile-up region has been observed in Comets Hyakutake and Hale-Bopp (Bouchez et al. 1999). For reviews on the interaction, see Coates (1997) and Rauer (1999).

Disconnection events (DEs) Work on the most spectacular plasma phenomenon, the DE, continues. Yi et al. (1996) developed a time-dependent magnetohydrodynamic code to simulate a comet crossing the heliospheric current sheet (HCS). An approximation was used to allow for the small thickness of the HCS when it is compressed as part of the interaction. The results of this simulation are that DEs are produced when a comet crosses the HCS, but not by another common feature in the solar wind. This conclusion was criticized by Wegmann (1998), which in turn was answered by Yi et al. (1998).

Observationally, the extensive coverage of DEs in Comet Halley (1985-1986) was utilized by Brandt et al. (1999). To minimize the uncertainties in single events, all $19 \mathrm{DEs}$ were analyzed as a group. The $19 \mathrm{DEs}$ are associated with HCS crossings, and no other property of the solar wind shows a one-to-one association.

The current situation is that there is considerable evidence for the HCS crossing and magnetic reconnection model of DEs, but not universal agreement.

Latitudinal structure In situ results from the Ulysses spacecraft have established that the equatorial and polar solar-wind environments for comets are very different (e.g., Phillips $1995 \mathrm{a}, \mathrm{b}$ ). At polar latitudes, the solar-wind speed is about $750 \mathrm{~km} / \mathrm{s}$, the density is about $3 \mathrm{~cm}^{-3}$, changes in properties are small and the HCS is not seen. At equatorial latitudes, the average solar-wind speed is about $450 \mathrm{~km} / \mathrm{s}$, the average density is about $9 \mathrm{~cm}^{-3}$, the HCS is seen, and changes in properties can be quite large. 
These differing environments should be seen in plasma tail properties. The tail orientation should reflect the solar-wind speed, the appearance should be less disturbed in the polar region, and DEs should occur in the equatorial region (assuming the HCS association discussed above).

This expectation seems to be confirmed by Comets de Vico in 1995, Hyakutake in 1996, and Hale-Bopp in 1997. Detailed results for Comet de Vico have been published (Brandt et al. 1997).

\subsection{Special topics}

$X$-ray emissions $\mathrm{X}$-ray $(0.09-2.0 \mathrm{keV})$ emission from a comet was first detected by ROSAT observations of C/Hyakutake (C/1996 B1) on March 26, 1996 (Lisse et al. 1996). The detected intensity $\left(4 \times 10^{15} \mathrm{erg} / \mathrm{s}\right)$ was more than one order of magnitude higher than predicted and about 70 times higher than the upper limit obtained from observations of C/Bradfield (1979 X) with the EINSTEIN satellite (Hudson et al. 1981). X-ray emission turned out to be a general property of comets. The emission was successfully found in four comets after a search in the ROSAT all sky survey archive (Dennerl et al. 1997). Soft X-ray emission was also detected by EUVE observations (Mumma et al. 1997; Krasnopolsky et al. 1997) and with the BEPPO-SAX satellite (Owens et al. 1998).

Among all the possible mechanisms of X-ray production only three are efficient enough to produce the observed steady state intensity (Krasnopolsky 1997; Wegmann et al. 1998): (1) Bremsstrahlung of energetic electrons by cometary gas (Bingham et al. 1997; Northrop et al. 1997; Uchida et al. 1998; Shapiro et al. 1999); (2) Charge exchange excitation of cometary gas by collisions with highly charged solar wind ions (Cravens 1997; Häberli et al. 1997a); and (3) Scattering of solar X-ray emission by very small $\left(10^{-19} \mathrm{~g}\right)$ particles (Utterback and Kissel 1990; Wickramasinghe and Hoyle 1996; Owen et al. 1998). The first two mechanisms can explain the emissions for most comets, even if some points need to be clarified. The last mechanism seems to work on very dusty comets, such as C/Hale-Bopp (Owens et al. 1998; Schulz et al. 1999).

Sodium tails On April 16, 1997, a third type of tail, composed only of neutral sodium atoms, was discovered in C/Hale-Bopp (Cremonese et al. 1997). It was clearly a tail distinct from the dust and ion tails. The first important result obtained from the analysis of the tail was the lifetime of the sodium atom for photoionization, as only the value of 47 hours, at $1 \mathrm{AU}$ from the Sun, could fit the sodium tail shape and the velocities measured along it (Cremonese et al. 1997). Such a value is three times larger than that adopted on different solar system objects in the last decades and was already suggested by Huebner et al. (1992) based on theoretical calculations. Soon after the discovery some teams realized that a $\mathrm{Na}$ tail was present also in their images (Wilson et al. 1998; Kupperman et al. 1998). However, further analyses of the data showed that $\mathrm{Na}$ atoms were also fully superimposed to the dust tail (Cremonese and Fulle 1999). In the Wilson data the narrow sodium tail was not visible because of the Swing effect acting on the sodium atoms, strongly modifying the tail appearance (Kawakita et al. 1998). Several authors suggested a distributed source in the coma to explain the origin of the narrow sodium tail, considering the nuclear source as minor. Combi et al. (1997) suggested a source related to the ions. This is not supported by the analysis of the spectra obtained in the coma (Arpigny et al. 1998; Brown et al. 1998; Rauer et al. 1998; Ip and Jorda 1998), suggesting instead dust particles or neutral molecules. Rietmeijer (1999) compares the cosmic and cometary $\mathrm{Na}$ content with the ones in IDPs and concludes that most of the $\mathrm{Na}$ in comets is contained in dust.

Hyakutake (C/1996 B2) C/Hyakutake passed unusually close to the Earth ( $\sim 0.1 \mathrm{AU})$ in March 1996, affording a unique opportunity for high spatial resolution observations. In addition, it was sufficiently bright that a wide range of techniques could be used to observe it, allowing for detection of molecules never before seen in a comet and details never before possible. Observations in the radio-, $\mathrm{mm}-$, and submm-wave regions proved powerful for 
probing parent species and isotope ratios. Transitions of $\mathrm{HCN}, \mathrm{H}^{13} \mathrm{CN}, \mathrm{HNC}, \mathrm{HNCO}, \mathrm{CO}$, $\mathrm{CH}_{3} \mathrm{OH}, \mathrm{H}_{2} \mathrm{CO}, \mathrm{OCS}, \mathrm{OH}, \mathrm{H}_{2} \mathrm{O}$, and other species were detected and mapped by a variety of telescopes (Bockelée-Morvan et al. 1998; Cosmovici et al. 1998; Gerard et al. 1998; Lis et al. 1997; Woodney et al. 1997; Wouterloot 1998). Deuterated species were detected to derive $\mathrm{D} / \mathrm{H}$ ratios in several species. These ratios were higher than the terrestrial ratio (Meier et al. 1998a, b). High resolution spectra were obtained at several sites $(R=26,000-200,000)$. Such techniques allow for identification of new emission lines, determination of isotope ratios, determination of rotational temperatures, and determination of the outflow speeds of several species (Bouchez et al. 1999; Combi et al. 1999; Meier et al. 1998c; Morrison et al. 1997). UV observations provided only the second time that $S_{2}$ was detected in a comet (Laffont et al. 1998) and the detection of the Ly- $\alpha$ coma by the SOHO Swan instrument (Bertaux et al. 1998). Many new species were detected in the IR, while higher spectral resolution allowed for resolving blended features. Notesco et al. (1997) showed that the $\mathrm{CO} / \mathrm{H}_{2} \mathrm{O}, \mathrm{CH}_{4} / \mathrm{H}_{2} \mathrm{O}$, and $\mathrm{C}_{2} \mathrm{H}_{6} / \mathrm{H}_{2} \mathrm{O}$ ratios found by Mumma et al. (1996) could be explained by trapping of these gases in amorphous water ice formed at $64-66 \mathrm{~K}$.

The dust activity of the comet during 23-25 March 1996 was observed by Schleicher et al. (1998) and a rotation period of $6.23 \mathrm{~h}$ was estimated from the regular dust puffs emitted by the nucleus. Imaging polarimetry and photometry of the comet was measured by Kiselev and Velichko (1998) and by Tozzi et al. (1997). Mason et al. (1998) observed the thermal dust emission and silicate band emission in the near- to mid-IR and identified abrupt changes in the dominating grain populations for this wavelength range.

The dust release and properties were modeled from dust tail observations of the comet by Fulle et al. (1997b). Sarmecanic et al. (1997) modeled the IR spectra observed in March 1996 by amorphous olivine and organic residue mixture.

Hale-Bopp (C/1995 O1) C/Hale-Bopp was the brightest comet of the past two decades. Perihelion was on 1 April 1997 at $r=0.9 \mathrm{AU}$. This, coupled with advances in detectors and a long lead time from discovery to perihelion, afforded an excellent opportunity to study the coma. Many results were presented at the Symposium on C/Hale-Bopp in Tenerife in January 1998 (proceedings in press in Earth Moon Planets).

Radio-, mm- and submm-wave observations were used to map out the sequence of release of species, to detect new species, and to determine isotope ratios (Bird et al. 1997; Biver et al. 1997; De Pater et al. 1998; Galt et al. 1998; Lovell et al. 1998; Meier et al. 1998a, b; Wright et al. 1998). It was shown that at large heliocentric distances, CO release dominates over $\mathrm{H}_{2} \mathrm{O}$ release. IR from the ground and from ISO proved particularly powerful for studies of the comet (Crovisier et al. 1997; Lellouch et al. 1998). Dello Russo et al. (1998) used high resolution IR spectra to detect OCS and suggested that some amount of OCS is released from a distributed source. The optical properties of the coma were mapped by many teams with techniques ranging from imaging through narrowband filters to high spectral resolution. The release of $\mathrm{H}_{2} \mathrm{O}$ was shown to be extremely high (at least $10 \times$ that of $\mathrm{C} /$ Halley) so that the collision-dominated zone was much larger than in most comets.

The dust coma structure and the colors were observed by Kidger et al. (1998). The distant activity of the comet beyond 6 AU pre-perihelion is modeled by Sekanina (1996). From the diurnal behavior of the pre-perihelion dust coma fan a first rotation model of the nucleus was deduced by Sekanina (1998). The 1996 appearance of the dust coma is described by Rauer et al. (1997). The porcupine jet pattern in the coma of this comet seen during 1996 was explained in the context of the embedded fan model (Sekanina and Boehnhardt 1997). Modeling of the dust haloes seen in C/Hale-Bopp in early 1997 led Sekanina (1998) to conclude the presence of two active nuclei. The spin period of the nucleus was estimated from the dust jet motion of the comet (Licandro et al. 1998). C/Hale-Bopp showed high polarization when compared to several other comets (Ganesh et al. 1998). Two different dust regions in the sunward and tailward side of the coma were proposed by Bellucci (1998) to explain the observed spatial differences in visible and near-IR continuum emission images. 
ISO observations in the far-IR suggest the presence of icy grains in the $10-\mu \mathrm{m}$ size range in the coma (Lellouch et al. 1998) at 2.9 AU pre-perihelion. Supporting these ISO results, Davies et al. (1997a) concluded from absorption features measured at 1.5 and $2.05 \mu \mathrm{m}$ in the comet that icy grains were present. Preliminary results from ISO spectroscopy indicate a color temperature of $162 \mathrm{~K}$ and the presence of silicate olivine emission features for the dust coma at 4.6 AU pre-perihelion (Crovisier et al. 1996). Near- and mid-IR spectroscopy in mid February 1997 by Williams et al. (1997) revealed that the light scattering and thermal emission in the dust coma was dominated by sub-micrometer sized grains. Using sub-mm imaging observations, Jewitt and Matthews (1999) detected large quantities of big dust. A detailed investigation of the silicate components in the dust coma was accomplished with mid-IR spectroscopy (Wooden et al. 1999). A search for diffuse interstellar bands with the HIRES spectrograph at Keck was unsuccessful (Herbig and McNally 1999). A first description and analysis of the dust tail striae is presented by Watanabe et al. (1997).

Comets that are planned to be visited by spacecraft There are now two comet missions in flight (DS-1 launched October 24, 1998, and Stardust launched February 7, 1999), two more approved (Contour for launch July 3, 2002, and Rosetta for launch January 21, 2003). There is the potential for visits of up to seven comets by these missions in the twelve years from 2001 to 2012 . The timeline for comet encounters by these missions is: 2001 Jan Wilson-Harrington flyby DS-1 (tentative), 2001 July Borrelly flyby DS-1 (tentative), 2003 Nov 12 Encke flyby Contour, 2004 Jan 2 Wild 2 flyby Stardust, 2006 Jan 15 Wild 2 sample return Stardust, 2006 June 18 SW-3 flyby Contour, 2008 Aug 16 D'Arrest flyby Contour, 2011 Nov Wirtanen orbit insertion Rosetta orbiter, 2012 July Wirtanen landing Rosetta lander.

The flybys of Wilson-Harrington and Borrelly by DS-1 require approval of additional funding after flyby of its primary target asteroid 1992 KD in July 1999. DS-1 is primarily a space technology flight test. Stardust will fly through the coma of $81 \mathrm{P} /$ Wild 2 to capture and return samples of the refractory dust component of the comet's coma. The spacecraft will also image the nucleus and coma, measure dust fluence, and conduct in-situ analysis of comet dust. Contour is a multiple flyby mission to three diverse comets. It could also be retargeted to a new comet if one is discovered during the mission. Contour carries a camera, mapping spectrometer, neutral and ion mass spectrometer, and a comet impact dust analyzer.

The Rosetta Orbiter carries an imager, vis-IR spectral mapper, UV spectrometer, millimeter spectrometer, neutral/ion mass spectrometer, dust impact mass spectrometer, gas chromatograph, atomic force microscope, dust flux monitor, plasma package, radio science investigation, and radio tomographic sounder. The Rosetta Lander carries a sample acquisition system, imaging system, $\alpha$-proton-xray spectrometer, evolved gas analyzer, magnetometer/plasma monitor, surface electrical/acoustic monitor, surface/subsurface science sensor, and the complementary instrument for tomographic sounding with the orbiter.

\author{
V. Zappalá \\ President of the Commission
}

